\title{
STUDY OF CLINICO-RADIOLOGICAL PARAMETERS IN DIAGNOSIS OF PEDIATRIC TUBERCULOSIS AT TERTIARY CARE HOSPITAL
}

\section{Respiratory Medicine}

Dr. Bhanu Desai

Associate Professor, Department Of Pediatrics, GCS Medical College, Hospital And Research Centre, Opposite DRM office, Naroda road, Ahmedabad-380025.

Dr Rushi Patel

Associate Professor, Department of Respiratory Medicine, GCS medical college, hospital and research centre, Opposite DRM office, Naroda road, Ahmedabad-380025.

\section{Dr. Yashvi Vijaykumar Dattani*

Dr. Tamannaben
Rameshbhai
Ninama

Amay Himanshu Khara Kanhai Harish Patel
Junior resident, Department of Pediatrics, GCS medical college, hospital and research centre, Opposite DRM office, Naroda road, Ahmedabad-380025.*Corresponding Author

Junior Resident, Department Of Pediatrics, GCS Medical College, Hospital And Research Centre, Opposite DRM Office, Naroda Road, Ahmedabad-380025.

Intern, GCS Medical College, Hospital and Research Centre, Opposite DRM Office, Naroda Road, Ahmedabad-380025

Intern, GCS Medical College, Hospital and Research Centre, Opposite DRM Office, Naroda Road, Ahmedabad-380025.

\begin{abstract}
Background: Global TB burden in India in children is about 2.2 lakh per year, among which pulmonary TB is most common presentation. Childhood TB contributes to about $10-20 \%$ of all TB in high burden countries and accounting for $8-20 \%$ TB related deaths. Children up to 14 years constitute about $35 \%$ of the population in our country and are expected to contribute about $10 \%$ of the case load. In order to achieve 'Zero death by TB', additional focus has to be directed towards TB in children. Paediatric clinical feature is variable in tuberculosis. Many cases of primary TB infection in children are asymptomatic and remain completely unnoticed or accidentally discovered at a later stage. In this study we looked for clinical and radiological presentation of childhood TB and its correlation in diagnosis within a period of 1 year in a tertiary care hospital. Methods: Total 254 children suspected of having tuberculosis were enrolled in the study and various microbiological, laboratory and radiological tests were used to diagnose the patients. Clinical samples were processed using standardized protocols. CBNAAT was the initial test of choice for microbiological confirmation.Results: Among 254 children, 69(27\%) had pulmonary tuberculosis, extrapulmonary tuberculosis was found in $76(30 \%)$ children. Fever was the most common (in 100\% cases) symptom in pulmonary TB. Most common radiological presentation was segmental consolidation (44\%). Amongst 69 pulmonary TB patients, 16(23\%) were Rifampicin resistant by CBNAAT method.Conclusion: Primary childhood TB being paucibacillary, makes microbiological diagnosis (gold standard) difficult. In relevant clinical setting, certain radiological lesions may be highly suggestive of tuberculosis: miliary pattern, hilar and/or paratracheal lymphadenopathy with or without parenchymal involvement and fibro-cavitatory lesions. All presumptive TB cases with these radiological patterns are considered to be probable TB and should be subjected to microbiological testing to confirm the diagnosis.
\end{abstract}

\section{KEYWORDS}

Childhood TB, CBNAAT, Radiological

\section{INTRODUCTION:}

Global TB report 2018 reports that in India, an estimated 2.2 lakh children become ill with tuberculosis (TB) each year (22\% of global TB burden) ${ }^{1}$, with a slightly higher burden among males. Pulmonary $\mathrm{TB}$ is the most common form in children. It is also known that about $10 \%$ of the cases reported to RNTCP are from children under 14 years of age. Adults and older children more often have the infectious form of TB which can be detected by testing of sputum while in general, children have forms of TB which may be more easily picked up on chest radiograph. Also, there is difficulty to access the specimen as children would more often swallow then bring up sputum and lower sensitivity of the microbiological tests in children further add to this difficulty.

Mycobacterium perhaps is one singular organism contributing to most death of under-five among world's middle \& lower-income nations, but it does not find place in the list of causes of death as it is difficult to diagnose and there is a large detection gap. Exact contribution by TB to 'Under 5 Mortality' is unknown. Many TB related deaths are possibly reported as pneumonia deaths due to similar respiratory symptoms. It is estimated that childhood TB constitutes $10-20 \%$ of all TB in high burden countries ${ }^{2}$, accounting for $8-20 \%$ of TB-related death $\mathrm{s}^{3-5}$.

Lack of standard definitions, absence of simple/reliable diagnostic test and more extrapulmonary TB cases add to lack of data on paediatric TB. There is also gross under estimation of magnitude of problem.

Countries are reporting on average $7 \%$ cases of TB among children, mostly clinically diagnosed and extrapulmonary. Numbers may be low as many children are treated outside National TB programmes. TB is usually an airborne infection. It is also to be emphasized that children can be infectious as well; especially older children and adolescents.

An imbalance between mycobacterial virulence and host immunity determines the progress of infection/disease. It is the interaction of both these factors that would decide whether the contacts would have an infection, whether the infection would remain latent or lead to a primary disease and as the child grows older, reactivation of the latent infection.

Risk of progression of the TB infection to disease is about $5-10 \%$ over lifetime in a healthy individual but this is higher in young children, nearly $>50 \%$ in infants and $25 \%$ in $1-5$ years. Rapid progression can occur in presence of severe malnutrition or HIV-TB co-infection, etc. Younger children are the most vulnerable and can have a disseminated disease: Tubercular meningitis (TBM), miliary, or a lymph node disease with complications. The school-going age is the safest period. Thereafter, the risk would again increase as the second peak appears at adolescence. They would have more of adult type disease and pleural effusions.

Presumptive Paediatric TB: refers to children with persistent fever and/or cough for more than 2 weeks, loss of weight/no weight gain (History of unexplained weight loss or no weight gain in past 3 months; loss of weight is defined as loss of more than $5 \%$ body weight as compared to highest weight recorded in last 3 months) and/ or 
history of contact with infectious TB cases (In a symptomatic child, contact with a person with any form of active TB within last 2 years may be significant).

Presumptive Extra Pulmonary TB: refers to the presence of organ specific symptoms and signs like swelling of lymph nodes, pain \& swelling in joints, neck stiffness, disorientation etc and/or constitutional symptoms like significant weight loss, persistent fever for $>2$ weeks, night sweats.

\section{AIMS AND OBJECTIVES:}

- To study different clinical presentation in paediatric tuberculosis.

- To study different radiological appearances in cases of paediatric tuberculosis.

- To study the clinico-radiological correlation in the diagnosis of paediatric $\mathrm{TB}$.

\section{MATERIALS AND METHODS:}

A total 254 Children $(<18$ years) presenting with suspected TB were prospectively enrolled by consecutive sampling from January 2019 to December 2019, at the inpatient and outpatient facilities of a tertiary care hospital, Ahmedabad. Suspected TB was defined as per the Indian Revised National TB Control Programme (RNTCP) guidelines ${ }^{6.7}$

Detailed history and physical examination were performed on each child by trained study physicians. Weight for age $Z$ scores (WAZ) were used to assess the nutritional status (WHO Anthro version 3.2.2, January 2011) with malnutrition defined as $\mathrm{WAZ}<2$. After appropriate counselling and consent, blood was drawn for HIV testing (positive on two separate HIV ELISA tests or HIV DNA PCR for children $<18$ months).

Gastric aspirates (up to 3 consecutive samples) and when indicated other appropriate clinical samples (lymph nodes, cerebrospinal fluid (CSF), pleural fluid, etc.) were obtained for mycobacterial culture. Chest radiographs were also performed on each child and Computed tomography (CT), magnetic resonance imaging (MRI), and ultrasonography (US) were performed as indicated. Clinical samples were processed using standardized protocols. CBNAAT was the initial test of choice for microbiological confirmation. A sample consisting of recently discharged material from the bronchial tree with minimum amount of oral or nasopharyngeal material, presence of mucoid or mucopurulent material and 2-5 $\mathrm{ml}$ in volume was collected in a sterile container after rinsing of the oral cavity with clean water. In case the patient did not spontaneously expectorate, any other available respiratory specimen like Gastric lavage/ Induced sputum/ bronchoalveolar lavage was collected and sample was subjected to CBNAAT.

Decontamination was performed with $\mathrm{N}$-acetyl-L-cysteine and sodium hydroxide, and the resuspended pellets were used for acid fast bacilli (AFB) staining and smear microscopy as well as inoculation of Lowenstein Jensen (LJ) slants. The remaining was cultured using the MGIT and incubated for at least 6 weeks. Positive cultures were confirmed by p-nitrobenzoic acid (PNB) on the MGIT 960. Drug susceptibility testing (DST) for isoniazid and rifampin was performed and confirmed on solid media. As a gold standard for diagnosis and for phenotypic DST, liquid cultures like Mycobacterium Growth Indicator tube ${ }^{\mathrm{TM}}$ (MGIT) cultures were used. Cultures were sent in all children who were CBNAAT negative and all presumptive DR TB cases.

\section{RESULTS AND DISCUSSION:}

In our study total 254 children with suspected TB were enrolled. Among them 69(27\%) had pulmonary tuberculosis, extrapulmonary tuberculosis was found in $76(30 \%)$ children. Age ranged from 0-18 years. Majority of the cases (42\%) were within 5-10 years. Based on the nutritional status $60 \%$ children had malnutrition. $68 \%$ patient of this series came from poor socioeconomic condition. Poor socioeconomic condition leads to malnutrition which in turn leads to immunodeficiency that predisposes to tuberculosis. Majority of the patients $52 \%$ presented with symptoms of $1-3$ month. Among them majority presented with constitutional features like fever, cough, weight loss and loss of appetite (Table-1)

Table-1: Distribution of patients by presenting complaint:

\begin{tabular}{|l|l|}
\hline Presenting complaints & Number of patients (\%) \\
\hline Fever & $69(100 \%)$ \\
\hline Cough & $49(72 \%)$ \\
\hline Weight loss & $33(48 \%)$ \\
\hline Loss of appetite & $20(28 \%)$ \\
\hline
\end{tabular}

\begin{tabular}{|l|l|}
\hline Respiratory distress & $15(21 \%)$ \\
\hline Altered sensorium & $10(13 \%)$ \\
\hline Convulsions & $10(13 \%)$ \\
\hline
\end{tabular}

Fever was reported to be $100 \%$ in this study. Every case of active pulmonary tuberculosis exhibits some degree of pyrexia, which is one of the important clinical criteria of activity. This is concordant with Singh $\mathrm{J}$ et $\mathrm{al}^{6}$ and Akbar MS et $\mathrm{al}^{7}$.Cough was the second most complaint in this study $(72 \%)$. About $48 \%$ of our patients reported loss of weight and $28 \%$ had loss of appetite.

Difficulty in breathing was observed in $21 \%$ of cases in our study. Cases with extrapulmonary tuberculosis presented most commonly with features of CNS involvement in form of altered sensorium(13\%) and convulsions $(13 \%$ ) each. In the investigation findings, about $56 \%$ patients had mild to moderate anaemia which was probably due to malnutrition. $80 \%$ patients had raised ESR. Demonstration of AFB is the gold standard for diagnosis of pulmonary tuberculosis which is usually not possible in case of children due to their failure to produce sputum. Thus the diagnosis of tuberculosis in children is mostly clinical and radiological where there is suspicion of tuberculosis.

TB can affect almost any organ system of the body. The clinical manifestations of extra- pulmonary tuberculosis (EPTB) are often non-specific. Imaging findings are also not always very specific thus making the diagnosis of EPTB more challenging than pulmonary tuberculosis. Several factors can lead to errors in TB diagnosis on chest radiology leading to both under \& over diagnosis. Issues associated with chest radiology includes quality of films, technical issues, inter and intraindividual variations, and artefact thymic shadow etc. Pulmonary tuberculosis produces a broad spectrum of radiological abnormalities. In our study we found calcified shadow in $7 \%$, segmental consolidation in $44 \%$, hilar lymphadenopathy in $32 \%$ and pleural effusion in $10 \%$ among the chest radiographs.

Table-2: Distribution of the patients on $x$-ray chest finding:

\begin{tabular}{|l|l|}
\hline X-ray findings & Number of patients (\%) \\
\hline Segmental consolidation & $30(44 \%)$ \\
\hline Hilar lymphadenopathy & $22(32 \%)$ \\
\hline Pleural effusion & $7(10 \%)$ \\
\hline Calcified shadow & $5(7 \%)$ \\
\hline Normal chest X-ray & $2(3 \%)$ \\
\hline
\end{tabular}

In the latter phase of the disease common abnormalities include exudative parenchymal densities predominantly in the apical and posterior segments of the upper lobes, cavitations with bronchogenic spread of disease, marked fibrotic response in the lungs, pleural effusion, empyema and fibrosis. Among 69 confirmed pulmonary tuberculosis $53(77 \%)$ were rifampicin sensitive and $16(23 \%)$ were rifampicin resistant based on the CBNAAT reports.

\section{DISCUSSION:}

Primary childhood TB being paucibacillary, makes microbiological diagnosis (gold standard) difficult. Hence, chest radiograph is an important tool to support the diagnosis of pulmonary TB in children in the absence of microbiological confirmation. In relevant clinical setting, certain radiological lesions may be highly suggestive of tuberculosis: miliary pattern, hilar and/or paratracheal lymphadenopathy with or without parenchymal involvement and fibrocavitatory lesions. All presumptive TB cases with these radiological patterns are considered to be probable TB and should be subjected to microbiology to confirm the diagnosis. In case microbiological investigations are negative these children can be considered to be cases of clinically diagnosed tuberculosis (TB). Clinical and radiological presentation is variable in different pathological state of pulmonary tuberculosis. CBNAAT is a real-time PCR rapid technique for diagnosis of TB and detection of rifampicin resistance conferring mutations within 2 hours. CBNAAT can be performed on both respiratory and non-respiratory specimens (GA, BAL, IS, pleural fluid, CSF, lymph node aspirate etc). The sensitivity and specificity of CBNAAT in sputum samples is around $98 \%$ and $99 \%$ for smear positive patients and $72 \%$ for smear negative culture positive patients. The sensitivity and specificity on GA have been $68 \%$ and $99 \%$ respectively. The yield of the test falls significantly to under $10 \%$, if it is offered purely on clinical basis (symptom basis). While it performs well with CSF and LN aspirates, its yield is very poor with pleural or ascitic fluid. Limited cases may not represent the actual clinical and radiological presentation of childhood pulmonary tuberculosis but 
definitely it gives as an idea about the presentation of pulmonary tuberculosis in the context of our country.

\section{CONCLUSION:}

In our study, extrapulmonary tuberculosis patients were more common than pulmonary tuberculosis. Most common age group affected was between 5 to 10 years. Lower socio-economic status and malnutrition were the most common contributing factors to tuberculosis. Fever followed by cough was the most common presenting complaint. Among the radiological findings segmental consolidation followed by hilar lymphadenopathy were the most common findings.

Among 69 confirmed pulmonary tuberculosis 53(77\%) were rifampicin sensitive based on the CBNAAT reports.

\section{Acknowledgements:}

We thank Respiratory Medicine Department and DOTS centre GCS Medical college, hospital and research centre, Ahmedabad.

Amay Himanshu Khara. GCS medical college, hospital and research centre G2 block, PG Hostel, GCS Medical college, hospital and research centre, opposite DRM office, Naroda road, Ahmedabad380025.

Kanhai Harish Patel. GCS medical college, hospital and research centre G2 block, PG Hostel, GCS Medical college, hospital and research centre, opposite DRM office, Naroda road, Ahmedabad380025 .

\section{REFERENCES:}

1) RNTCP Updated Paediatric TB Guidelines 2019 Developed by REVISED NATIONAL TUBERCULOSIS CONTROL PROGRAM and INDIAN ACADEMY OF PEDIATRICS

2) B. J. Marais, A. C. Hesseling, R. P. Gie, H. S. Schaaf, and N. Beyers, "The burden of childhood tuberculosis and the accuracy of community-based surveillance data," International Journal of Tuberculosis and Lung Disease, vol. 10, no. 3, pp. 259-263, International Journal of Tuberculosis and Lung Disease, vol. 10, no. 3, pp. 259-263, 2006

3) S. K. Kabra, R. Lodha, and V. Seth, "Tuberculosis in children: what has changed in last 20 years?”'Indian Journal of Paediatrics, vol. 69, supplement 1, pp. S5-S10, 2002.

4) S. Swaminathan and B. Rekha, "Pediatric tuberculosis: global overview and challenges,"Clinical Infectious Diseases, vol. 50, supplement 3, pp. S184-S194, 2010.

5) C. M. Perez-Velez and B. J. Marais, "Tuberculosis in children," The New England Journal of Medicine, vol. 367, no. 4, pp. 348-361, 2012.

6) Singh J. Sociomedical study of behavior and symptoms of TB patient's paper presented at 30 th National Conference on TB and Chest Diseases Hyderabad 1975 .

7) Akbar MS. Childhood tuberculosis clinical experience at Dhaka Shihsu Hospital, multidisciplinary seminar on tuberculosis, Dhaka, Society of Surgeons of Bangladesh, Oct 1990:39. 